\title{
Chemical Abundance Patterns of the Galaxy's Thick Disk Population
}

\author{
Mary-Margaret Brewer ${ }^{1}$ and Bruce W. Carney ${ }^{1,2}$ \\ ${ }^{1}$ Department of Physics and Astronomy, University of North Carolina, Chapel Hill, NC 27599-3255, USA \\ ${ }^{2}$ E-mail: bruce@ physics.unc.edu
}

Received 2003 September 24, accepted 2004 March 9

\begin{abstract}
We discuss our work on chemical abundance ratios of stars belonging to the thick-disk and thin-disk stellar populations. We discuss the selection of stars, and show that the two samples of stars have different $[\alpha / \mathrm{Fe}]$ versus $[\mathrm{Fe} / \mathrm{H}]$ behaviour.
\end{abstract}

Keywords: Galaxy: abundances — Galaxy: disk — Galaxy: evolution

\section{Introduction}

On the basis of star counts, Gilmore \& Reid (1983) argued that the Galaxy contains a second disk population, with a larger vertical scale distribution and, presumably, hotter kinematics than the 'classical old disk'. This 'thick disk', as it is now commonly called, is of considerable relevance to the understanding of the evolution of our Galaxy and, by extension, other disk galaxies for either or both of two reasons. First, the formation and evolution of disk galaxies are not well understood. For example, Burkert et al. (1992) argued that the thick disk was a precursor to the 'old thin disk' and thus a thick disk is a natural early step in the chemodynamical evolution of galactic disks. Others, including Carney et al. (1989), argued that the thick disk is too distinct in its chemodynamical behaviour, manifested, for example, by $[\mathrm{Fe} / \mathrm{H}]$ versus $V$ velocity, to share a common origin with the thin disk. In this case, the thick disk is likely to have arisen from a merger event at some point in the (possibly distant) past. In view of the merging Sagittarius dwarf galaxy, and the predictions of some cosmological models for a 'bottom-up' formation of galaxies from large numbers of mergers, the origin of the thick disk is especially relevant.

So how do we further test if the thick disk is simply the older stages of the formation of the Galaxy's thin disk, or whether it represents a truly separate population, with a separate history? By 'population', we mean here an ensemble of gas and stars that share a coherent history. Alas, we have no means to probe the gaseous component of the thick disk since it has presumably been absorbed by and mixed within the more massive thin disk. Note that the low metallicity of the thick disk $\langle[\mathrm{Fe} / \mathrm{H}]\rangle \approx-0.5$ to -0.7 (Gilmore \& Wyse 1985; Carney et al. 1989) implies that considerable gas must have been lost, at least in a simple model where the yield of heavy elements is the same as it has been in the thin disk. In other words, had the thick disk proceeded to transform gas into stars, its metallicity should be comparable to that of the thin disk. Like the halo, but to a lesser degree, the thick disk probably has a low metallicity because its gas was stripped away before the mean metallicity level had risen to near-solar levels. However, the residual stars retain considerable dynamical and chemical information that enable us to probe the history and relationships of the disk stars, if we can determine a means to assign probable membership of stars to the thin disk or thick-disk populations.

Aside from simple metallicity versus kinematics, stars retain the history of star formation in their abundance patterns. An ensemble of gas and stars should initially yield stars whose abundance patterns reflect the ejecta from Type II supernovae, and if star formation is slow enough for the ejecta of Type Ia supernovae to be incorporated into gas undergoing star formation, its signature should appear at the mean metallicity the interstellar medium and newly forming stars had reached. This is taken to be the basic explanation for the enhanced abundances of ' $\alpha$ ' elements $(\mathrm{O}, \mathrm{Mg}, \mathrm{Si}, \mathrm{Ca}, \mathrm{Ti})$ in the solar neighbourhood at the lowest metallicities, and the steady decline in $[\alpha / \mathrm{Fe}]$ abundances beginning at $[\mathrm{Fe} / \mathrm{H}] \approx-1.0$ and continuing to higher metallicity. In other words, the solar neighbourhood stars had reached a mean metallicity of $[\mathrm{Fe} / \mathrm{H}] \approx-1$ when the Type Ia supernovae began to significantly enrich the interstellar medium. Thus one possible test of the interrelationship between thin-disk and thick-disk populations is to ask, simply, whether they share a common $[\alpha / \mathrm{Fe}]$ versus $[\mathrm{Fe} / \mathrm{H}]$ relation. If they do, the common history model is strengthened (though hardly proven). If they do not, then one must either conclude that the thick disk evolved independently of the thin disk or that the chemical enrichment models of the joint thick disk-thin disk evolution are rather more complicated (perhaps by the unaccounted-for lost gas?).

Several studies have been undertaken to explore the [element $/ \mathrm{Fe}]$ versus $[\mathrm{Fe} / \mathrm{H}]$ histories of the thin-disk versus thick-disk stars, including Fuhrmann (1998), Chen et al. (2000), Prochaska et al. (2000), and Feltzing et al. (2003). We do not feel that the first two studies employed samples optimally chosen to explore the question of the 
(a)

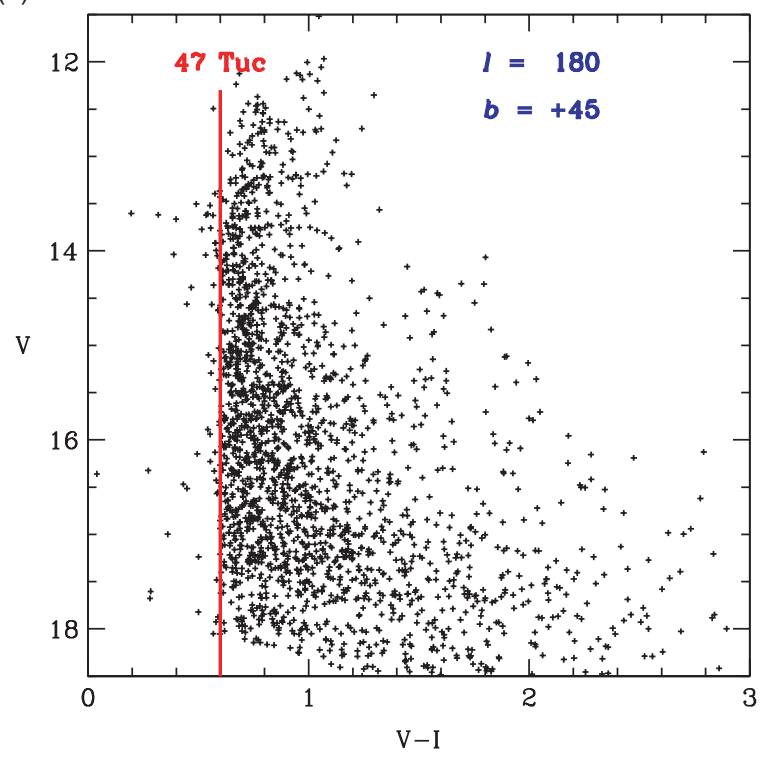

(b)

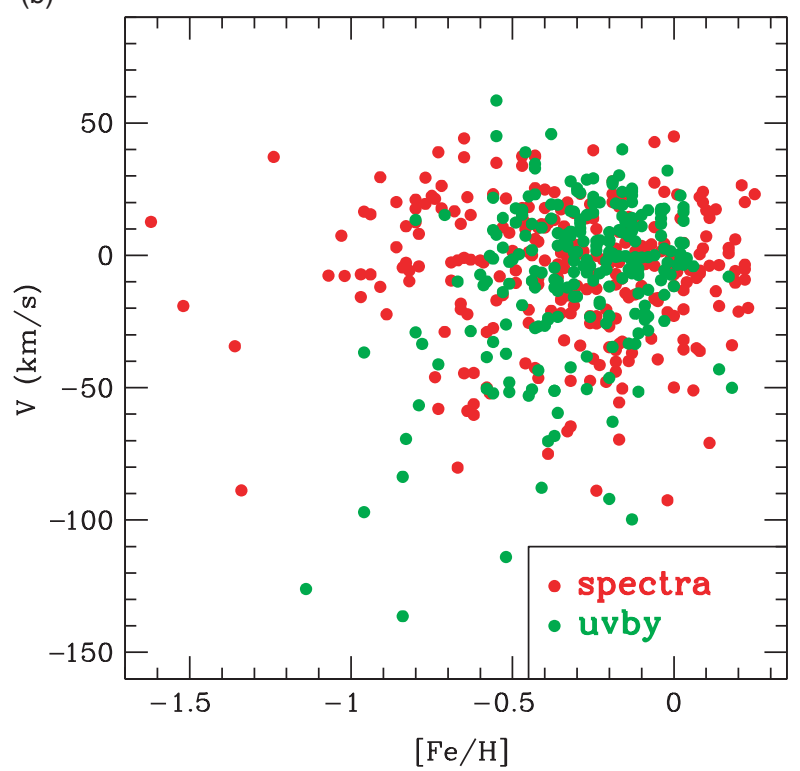

Figure 1 (a) A sample high-latitude field in the direction of the Galactic anti-centre region, covering several square degrees. The colour of the de-reddened main sequence turn-off of the intermediate metallicity globular cluster 47 Tuc is superposed. (b) Galactic $V$ velocities versus $[\mathrm{Fe} / \mathrm{H}]$ for a sample of stars selected without kinematic bias, and whose estimated life expectancies exceed the age of the Galaxy.

origin of the thick disk, and we report here on preliminary results of a new study aimed at correcting these difficulties. Two problems are most common. First, selection of stars has often been made with no attention to the stellar ages or their life expectancies. If the thick-disk stars are uniformly old, then any star whose life expectancy is brief will necessarily belong to the thin disk, regardless of its kinematics or metallicity. In this case one would be sampling the tails of the thin disk's velocity or metallicity distributions - useful information, to be sure, about the evolution of the thin disk, but not obviously relevant to the origin of the thick disk. In our opinion, this problem affects the stars selected for study by Fuhrmann (1998) and Chen et al. (2000). Since Feltzing et al. (2003) selected their stars by kinematics alone, this problem may or may not exist for their sample. In the absence of reliable age estimates for individual stars, Prochaska et al. (2000) selected only cooler dwarfs with long life expectancies, but their sample demonstrated a second problem: they only studied stars thought to belong to the thick disk, and a comparison was made to thin-disk stars only via the published literature, especially the results of Edvardsson et al. (1993). Modest but systematic differences in assignment of temperatures and other aspects of the abundance analyses can compromise the abundance pattern comparisons in such cases.

Is the thick disk uniformly old? Dinescu et al. (1999) have computed space velocities for a number of globular clusters, finding a number of them have disk-like kinematics. Some of these also have age determinations relative to globular clusters with halo kinematics. NGC $6752([\mathrm{Fe} / \mathrm{H}]=-1.5)$ is a disk cluster with an age indistinguishably different from halo clusters (Rosenberg et al. 1999; Salaris \& Weiss 2002), while the more metal-rich disk clusters $47 \mathrm{Tuc}([\mathrm{Fe} / \mathrm{H}]=-0.7)$ and M71 $([\mathrm{Fe} / \mathrm{H}]=-0.6)$ may be slightly younger, but still very old. Three globular clusters are hardly representative, but consider Figure 1(a), which is a colour-magnitude diagram of a high-latitude field. Note the sharpness of the blue edge to the colour distributions at almost all magnitudes. This is presumably the main sequence turn-off seen at increasing distances from mid-plane at fainter magnitudes. Since 47 Tuc has a metallicity similar to that of the field thick-disk stars, we have superposed the de-reddened colour of its main sequence turn-off on the Figure. The simplest conclusion is that there is little evidence for younger stars at distances from the plane at which the thick disk is the dominant contributor.

\section{Target Selection and Observations}

We have selected our targets for abundance determinations following a study by Naumov (1999) of the Galaxy's thindisk and thick-disk stellar populations. Naumov undertook an exhaustive review of available $u v b y$ photometry and parallax data and selected dwarfs generally lying within $60 \mathrm{pc}$ of the Sun. Temperatures were determined using the colour-temperature calibrations of Alonso et al. (1996), and metallicities were estimated using the calibrations of Schuster \& Nissen (1989). The sample was then trimmed of all stars whose temperatures and metallicities indicated life expectancies of less than $10 \mathrm{Gyr}$. A second sample of cool dwarfs was identified via objective prism spectroscopy in three cardinal directions near $b \approx 0^{\circ}$ : $l \approx 0,90,180^{\circ}$. A series of spectral indices defined by Rose (1984) were used to separate dwarfs from subgiants and giants, and then, again, only the cooler dwarfs were retained. For both samples, we then obtained high 
resolution $(R \approx 30000)$ but low $\mathrm{S} / \mathrm{N}(\approx 15)$ spectra covering $45 \AA$ centred near $\lambda 5200$ using the 1.5 -m telescopes of the Center for Astrophysics. Following the methods discussed by Carney et al. (1987) and Laird et al. (1988), mean metallicities were derived from all these spectra using $\chi^{2}$ matches to an extensive grid of synthetic spectra. The radial velocities of the in-plane sample relate directly to the $U V W$ velocities: $V_{\text {rad }}$ at $l \approx 90$ and $b \approx 0$ provides the $V$ velocity, for example. The metallicities of the ' $u v b y$ ' sample stars' spectra agreed very well with those estimated from the photometry, except for the occasional double-lined system. (We are now re-evaluating our more extensive collection of spectra for publication.) In Figure 1(b) we show the results of the derived metallicities, indicated by $[\mathrm{Fe} / \mathrm{H}]$, with the $V$ velocities. Note that most of the stars are centred around $V \approx 0 \mathrm{~km} \mathrm{~s}^{-1}$, with $[\mathrm{Fe} / \mathrm{H}]$ values ranging from about -1 to above solar. Note that the dispersion in $V$ barely reaches up to about $+50 \mathrm{~km} \mathrm{~s}^{-1}$, but well below $-50 \mathrm{~km} \mathrm{~s}^{-1}$. This 'tail' to more negative $V$ velocities is what we associate with the thick disk. Note, too, that the thick disk remains distinct from the dominant thin disk over the full range of metallicities, which is not consistent with simple models wherein the metal-poor thick disk evolves into the more metal-rich thin disk.

We selected our stars for study from Figure 1(b), and similar considerations of the $U$ and $W$ velocities. Thus, like many other studies, we have distinguished the thin- and thick-disk stars based on kinematics, but with the added provision that the stars must have very long life expectancies. Because the ' $u v b y$ ' sample stars were brighter than those identified from objective prism spectra, and all had measured proper motions (and many had measured parallaxes as well), we chose all 9 thin-disk and 14 thick-disk stars from the ' $u v b y$ ' sample.

All the stars were observed using two different echelle and cross-disperser gratings with the KPNO 4-m telescope. One grating setting was optimised for the blue and the other for the red. We obtained essentially complete wavelength coverage over $\lambda \lambda 3820-7400$, with a resolving power of 32000 and $S / N=80-200$ at the $\lambda 4129 \mathrm{Eu} \mathrm{II}$ line and 280-460 at the $\lambda 6645 \mathrm{Eu}$ II line.

\section{Results}

At the time of the meeting, we had not yet completed our abundance analyses of the $r$ - and s-process elements, and so we confine our results here to $[\alpha / \mathrm{Fe}]=$ $[\langle\mathrm{Mg}, \mathrm{Si}, \mathrm{Ca}, \mathrm{Ti}\rangle / \mathrm{Fe}]$, shown in Figure 2 . Thick-disk stars are shown as blue dots, and thin-disk stars as red dots. We have also included the results of Prochaska et al. (2000) as blue circles since they also restricted their analyses explicitly to stars with very long life expectancies. Finally, we include results from Edvardsson et al. (1993), but only for stars with ages that equal or exceed $10^{10}$ years. Blue squares are stars with $|W|>20 \mathrm{~km} \mathrm{~s}^{-1}$ and $-100 \leq V \leq-50 \mathrm{~km} \mathrm{~s}^{-1}$, while red squares are stars with thin-disk kinematics, $|W| \leq 20 \mathrm{~km} \mathrm{~s}^{-1}$ and $-20 \leq V \leq+20 \mathrm{~km} \mathrm{~s}^{-1}$.

We have also superposed crude representations of the trends for the thin-disk and thick-disk stars. They clearly

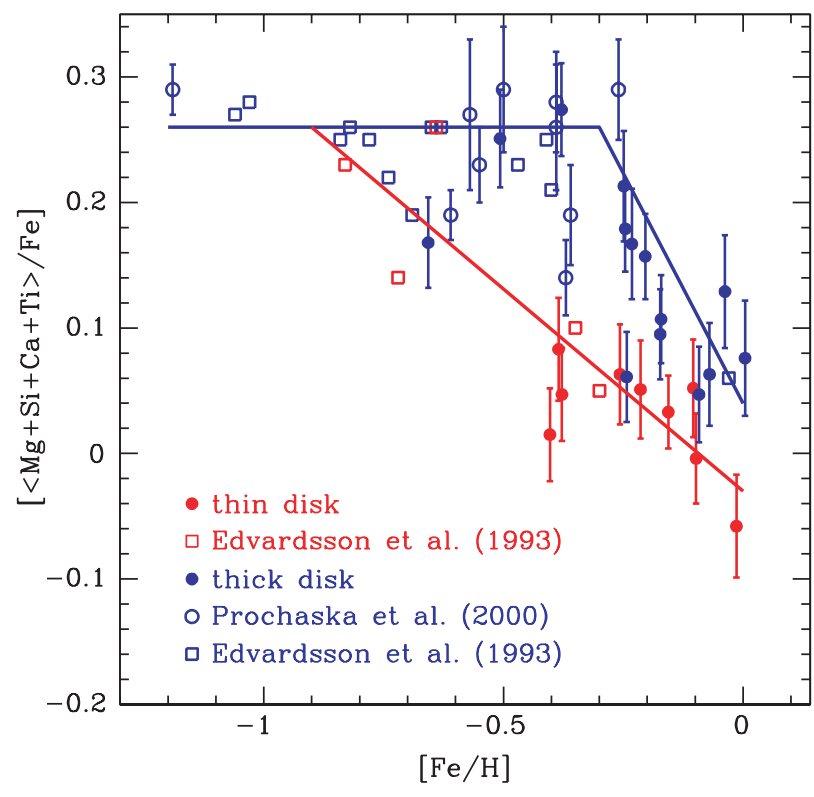

Figure 2 The mean abundances of the ' $\alpha$ ' elements relative to iron for the longest-lived or oldest stars associated with the thin disk (red) or the thick disk (blue), taken from our work (dots), Prochaska et al. (2000: circles), and Edvardsson et al. (1993: squares).

follow different trends. Both appear to have significantly enhanced $[\alpha / \mathrm{Fe}]$ ratios of close to +0.3 for $[\mathrm{Fe} / \mathrm{H}]<-1$. But the thin-disk chemical evolution led to declining values of $[\alpha / \mathrm{Fe}]$ as $[\mathrm{Fe} / \mathrm{H}]$ rose above that level, presumably due to Type Ia supernovae contributions to the interstellar medium, while the thick disk did not begin to reveal signs of Type Ia nucleosynthesis until $[\mathrm{Fe} / \mathrm{H}] \approx-0.3$.

The simplest interpretation of Figures 1(b) and 2 is that the thick disk had a chemical and dynamical evolution that was independent of the thin disk. We may further speculate that the following scenario can explain our observations. Before it merged with the Milky Way to create what we see now as the thick disk, the satellite galaxy's chemical evolution had proceeded rapidly, perhaps at a speed comparable to that seen in the Galaxy's halo, so that Type Ia supernovae contributions began to appear only when the gas content had reached a mean metallicity of $[\mathrm{Fe} / \mathrm{H}] \approx-0.3$. Star formation continued, however, since the thick disk's mean metallicity more than doubled, with $[\mathrm{Fe} / \mathrm{H}]$ reaching solar and higher levels. But even this proceeded relatively rapidly since the ages of the bulk of the thick-disk stars all appear to be great, certainly greater than the ages of the average thin-disk stars with comparable metallicities. Star formation in the thick disk presumably ceased in the final stages of the merger event, when any remaining gas was stripped away. The merger occurred with a significant angular momentum content, so that the collisionless stars only lag the thin-disk mean rotation rate by a few tens of kilometres per second in mid-plane. The victim's stars that were stripped at larger distances from the plane, however, may not have quite so high an angular momentum component (see Chiba \& Beers 2000; Gilmore et al. 2002).

Do any of the remaining satellite galaxies show such a chemical abundance pattern indicative of a similar 
chemical evolution history? We focus here only on systems that, like the thick disk, are composed mostly if not entirely of very old stars: the Draco and Ursa Minor dwarf galaxies. Their chemical abundance patterns (and presumably their chemical enrichment histories) show behaviour in $[\alpha / \mathrm{Fe}]$ versus $[\mathrm{Fe} / \mathrm{H}]$ that is very different from that of the thick disk. Shetrone et al. (2001) found solar-like (i.e. low) values of $[\alpha / \mathrm{Fe}]$ at even the lowest $[\mathrm{Fe} / \mathrm{H}]$ values in these two galaxies. The Galaxy's bulge population also appears to be different from the thick disk, especially when one considers the ' $\alpha$ ' elements in detail. McWilliam \& Rich (1994) found that $\mathrm{Mg}$ and $\mathrm{Ti}$ remain high $(\approx+0.4)$ for $[\mathrm{Fe} / \mathrm{H}]$ values as high as solar, while $\mathrm{Si}$ and $\mathrm{Ca}$ decline to solar values by $[\mathrm{Fe} / \mathrm{H}] \approx-0.3$. The thick-disk stars show elevated levels of all four elements for $[\mathrm{Fe} / \mathrm{H}] \leq-0.3$, above which they decline to solar values. Recently Pompéia et al. (2003) have reported abundances for over 30 local stars with kinematics that indicate kinship with the Galactic bulge population. Using the iron abundances they derived from only the neutral iron lines, as adopted by our work, Prochaska et al. (2000), and Edvardsson et al. (1993), we find that the $[(\mathrm{Mg}+\mathrm{Ti}) / \mathrm{Fe}]$ versus $[\mathrm{Fe} / \mathrm{H}]$ results of Pompéia et al. (2003) are broadly similar to the thindisk stars and unlike our thick-disk sample (and also unlike the results of the in situ bulge stars studied by McWilliam \& Rich 1994). Further, the results of Pompéia et al. (2003) for $[(\mathrm{Si}+\mathrm{Ca}) / \mathrm{Fe}]$ do not agree with any of these other studies. There remains the possibility that the differences are due to the methods of analysis rather than the stellar abundances themselves, and we have begun a more detailed comparison. But if the relative differences between our results and those of Edvardsson et al. (1993), McWilliam \& Rich (1994), and Pompéia et al. (2003) hold up, the latter may have identified a stellar group or population that has experienced a different chemical history than the thin-disk, thick-disk, or bulge populations.

Thus after a quick first look, there do not at this time appear to be any satellite systems with a chemical abundance history that we infer for the thick disk, but a lot of work remains to be done.

\section{Conclusions}

Using criteria that assure us of reasonable success in identifying local stars as belonging to the thin disk or the thick disk, we find that the two populations show very different behaviour in the trends of $[\alpha / \mathrm{Fe}]$ versus $[\mathrm{Fe} / \mathrm{H}]$, and we conclude that the simplest interpretation is that the two populations evolved separately, and in separate galaxies, until the parent galaxy of the thick disk was absorbed by the Milky Way.

\section{Acknowledgments}

We thank the US National Science Foundation for support of this work through grant AST-9988156.

\section{References}

Alonso, A., Arribas, S., \& Martínez-Roger, C. 1996, A\&A, 313, 873 Burkert, A., Truran, J. W., \& Hensler, G. 1992, ApJ, 391, 651

Carney, B. W., Latham, D. W., \& Laird, J. B. 1989, AJ, 97, 423

Carney, B. W., Laird, J. B., Latham, D. W., \& Kurucz, R. L. 1987, AJ, 94, 1066

Chen, Y. Q., Nissen, P. E., Zhao, G., Zhang, H. W., \& Benoni, T. 2000, A\&AS, 141, 491

Chiba, M., \& Beers, T. 2000, AJ, 119, 2843

Dinescu, D. I., Girard, T. M., \& van Altena, W. F. 1999, AJ, 117, 1792

Edvardsson, B., Andersen, J., Gustafsson, B., Lambert, D. L., Nissen, P. E., \& Tomkin, J. 1993, A\&A, 275, 101

Feltzing, S., Bensby, T., \& Lundström, I. 2003, A\&A, 397, L1

Fuhrmann, K. 1998, A\&A, 338, 161, 1998

Gilmore, G., \& Reid, I. N. 1983, MNRAS, 202, 1025

Gilmore, G., \& Wyse, R. F. G. 1985, AJ, 90, 2015

Gilmore, G., Wyse, R. F. G., \& Norris, J. 2002, ApJ, 574, L39

Laird, J. B., Carney, B. W., \& Latham, D. L. 1988, AJ, 95, 1843

McWilliam, A., \& Rich, R. M. 1994, ApJS, 91, 749

Naumov, S. 1999, PhD thesis, University of North Carolina at Chapel Hill

Pompéia, L., Barbuy, B., \& Grenon, M. 2003, ApJ, 592, 1173

Prochaska, J. X., Naumov, S. O., Carney, B. W., McWilliam, A., \& Wolfe, A. M. 2000, AJ, 120, 2513

Rose, J. A. 1984, AJ, 89, 1238

Rosenberg, A., Saviane, I., Piotto, G., \& Aparicio, A. 1999, AJ, 118,2306

Salaris, M., \& Weiss, A. 2002, A\&A, 388, 492

Schuster, W. J., \& Nissen, P. E. 1989, A\&A, 221, 65

Shetrone, M. D., Côté, P., \& Sargent, W. L. W. 2001, ApJ, 548, 592 